\title{
RECOLECCIÓN DE ESPECIES SILVESTRES DE PAPA EN GUATEMALA1
}

\author{
David M. Spooner ${ }^{2}$, Vicente Martinez ${ }^{3}$, Roel Hoekstra ${ }^{4}$, Ronald G. van den Berg 5
}

\begin{abstract}
RESUMEN
Recolección de especies silvestres de papa en Guatemala. El objetivo de este trabajo fue recolectar germoplasma de especies silvestres de papa, para su conservación, e incremento para ponerlas a disposición a nivel internacional, y reunir datos de campo para continuar con los estudios taxonómicos. De Solanum agrimonifolium se efectuaron 13 recolectas en los departamentos de Huehuetenango, San Marcos, Quetzaltenango, Totonicapan, Sololá y Chimaltenango en altitudes comprendidas de 2.340 a $3.300 \mathrm{msnm}$; de S. bulbocastanum subsp. partitum se obtuvieron seis recolectas en los departamentos de Huehuetenango y Baja Verapaz; de S. clarum se obtuvieron 17 recolectas en los departamentos de Huehuetenango, San Marcos, Sololá y Totonicapan, en altitudes de 3,000 a 3,500 msnm; de $S$. demissum solo se encontró una localidad en Totonicapan; y de S. morelliforme se obtuvieron seis recolectas de los departamentos de Huehuetenango, San Marcos y Totonicapan en alturas de 2.900 a 3.050 msnm. Los hábitats, donde estas especies crecen, están muy deteriorados por la deforestación y el cambio de uso del suelo. Se plantean dos áreas para la conservación in situ por la concentración de especies silvestres de papa y porque aún mantienen determinadas áreas casi naturales: 1) La Cumbre de María Tecún en el departamento de Totonicapan, y 2) áreas dispersas en la Sierra de los Cuchumatanes.
\end{abstract}

\begin{abstract}
Collecting of potato wild species in Guatemala. The objective of this collection o fpotatoes expedition was to collect germplasm of potatoes wild species for their conservation and regeneration to put them available to the international scientific community as well as to gather field data to carry on taxonomic studies. A total of accessions of five species was collected. Thirteen accessions of Solanum agrimonifolium were collected in the Huehuetenango, San Marcos, Quetzaltenango, Totonicapán, Sololá and Chimaltenango provinces located in altitudes ranging from 2,340 to 3,300 meters above sea level (masl); six accessions of S. bulbocastanum subsp. partitum were gotten in the Huehuetenango and Baja Verapaz provinces; 17 accessions of S. clarum were found in the Huehuetenango, San Marcos, Sololá, and Totonicapán provinces in altitudes extending from 3,000 to 3,500 masl; on1y one accession of S. demissum was found in Totonicapán province; and six accessions of $S$. morelliforme were gotten in Huehuetenango, San Marcos and Totonicapán provinces in altitudes ranging from 2,900 to 3,050 masl. The habitats where these species grow are very dist urbed due to deforestation and changes in the use of the soil. As a result, the guatemalan potato wild species are endangered species. It is notorious that in many locations where 50 years ago wild germplasm was gathered it no longer exist.
\end{abstract}

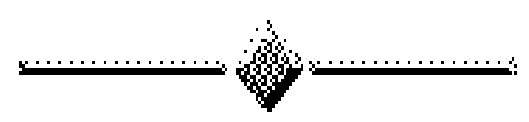

\section{INTRODUCCION}

Desde el suroeste de los Estados Unidos al sureste de Chile crece Solanum L. Seco Petota Dumort. De acuerdo con el último tratamiento taxonómico de Hawkes (1990), existen en siete especies cultivadas y 225 silvestres.

Muchas de las especies silvestres de papa están distribuidas en los Andes, pero cerca de 30 crecen en México y Centro América, con cinco en Guatemala: Solanum agrimonifolium Rydb., S. bulbocastanum Dunal, S. clarum Correll, S. demissum Lindl., y S. morellifor- me, Bitter y G. Muench (Correl, 1952, 1962; Gentry y Standley, 1974; Hawkes, 1990). Estas especies también crecen en México, y alcanzan su distribución más al sureste en Guatemala, excepto $S$. agrimonifolium, que adicionalmente crece en Honduras. Una sexta especie, S. oxycarpum Schiede, posiblemente también crece en Guatemala, pero involucra una confusión taxonómica con S. agrimonifolium.

La importancia de las especies silvestres de papa radica en que son fuente de resistencia o tolerancia a plagas, enfermedades y condiciones adversas del ambiente

1 Presentado en la XLII Reunión Anual del PCCMCA en El Salvador, Centroamérica, 1996.

2 Departamento de Horticultura de la Universidad de Winsconsin. Estados Unidos.

3 Instituto de Ciencia y Tecnología Agócolas (ICT A). Guatemala.

4 Centro de Recursos Genéticos. Holanda.

5 Departamento de Taxonomía de la Universidad Agócola de Wageningen. Holanda 
para las especies cultivadas, así en las especies silvestres presentes en Guatemala se ha encontrado resistencia al tizón tardío (Phytophthora infestans), a la podredumbre blanda (Erwinia carotovora), al virus y de la papa, al escarabajo colorado (Leptinotarsa decemlineata), a los Afidos (Myzus persicae y Macrosiphum euphorbiae), a los nematodos (Globodera rostochiensis y G. pallida), tolerancia al frío, calor y sequía (Hawkes, 1990).

Con equipos importantes se han llevado a cabo en Guatemala nueve recolecciones individuales (Cuadro 1). Türckheim, Skutch, Aguilar G., Standley, Steyermark, Roe, y Beaman han realizado viajes de recolección generales de especímenes para herbario y dentro de éstas han recolectado papas silvestres. Recolecciones específicas para papa han sido realizadas por Graham en México y Guatemala para obtener material para estudios del tizón tardío y relaciones de cruzamiento genético de las especies diploides de esta área (Graham, Neiderhauser, Servin, 1959; Graham y Dionne, 1961). Hawkes, Hjerting, y Lester recolectaron, como parte de una gran expedición de papa, desde el suroeste de Esta- dos Unidos hasta Nicaragua (Hawkes, 1959). Todo el germoplasma de papa silvestre de Guatemala de que se disponía hasta antes de este trabajo fue hecho por Graham, quien recolectó seis entradas de S. agrimonifolium, S. clarum y $S$. morelliforme; y Hawkes, quien hizo diez entradas de $S$. agrimonifolium, S. bulbocastanum, S. clarum, S. demissum y S. morelliforme.

Centroamérica es una región relativamente poco representada en germoplasma de papa silvestre. En 1995 en Guatemala se efectuó una recolección de papas silvestre conjuntamente con el Instituto de Ciencia y Tecnología Agrícolas (ICTA) de Guatemala; el Centre for Genetic Resources de Holanda (CGN); y el National Research SupportProgram-6 del Departamento de Agricultura de los Estados Unidos (NRSP-6). El objetivo de esta expedición fue recolectar germoplasma de las especies silvestres de papa existentes en Guatemala, para su conservación e incrementarlas rápidamente para ponerlas a libre disposición a nivel internacional, y reunir datos de campo para continuar con los estudios taxonómicos.

Cuadro 1. Recolectas de herbario y germoplasma de papas silvestres en Guatemala, previas a esta recolección.

\begin{tabular}{|c|c|c|c|}
\hline Recolector & Fechas & Especies & Departamentos \\
\hline Hans von Türckheim & jul., 1908 & S. bulbocastanum & Baja Verapaz \\
\hline Alexander F. Skutch & ago.3, 1933; 27, 1934 & S. clarum, S. morelliforme & Chimaltenango, Quiché \\
\hline José I. Aguilar & $1939-1943$ & S. bulbocastanum & Guatemala \\
\hline Paul C. Standley & $\begin{array}{l}\text { feb. 11-mar. } 131939 \\
\text { feb. 1-16 } 1941 \\
\text { mar. 13, } 1959\end{array}$ & $\begin{array}{l}\text { S. agrimonifolium, } \\
\text { S. demissum }\end{array}$ & $\begin{array}{l}\text { Quetzaltenango, Sacatepequez, } \\
\text { San Marcos }\end{array}$ \\
\hline Julián A. Steyermark & $\begin{array}{l}\text { ene. } 22,1940 \text {-sept. } \\
7,1942\end{array}$ & $\begin{array}{l}\text { S. agrimonifolium, S. clarum, } \\
\text { S. bulbocastanum, S. demissum, } \\
\text { S. morelliforme }\end{array}$ & $\begin{array}{l}\text { Huehuetenango, El Progreso, } \\
\text { Quetzaltenango, San Marcos, } \\
\text { Sololá }\end{array}$ \\
\hline Keith M. Graham & ago. 6-oct. 23, 1956 & $\begin{array}{l}\text { S. agrimonifolium, S. clarum, } \\
\text { S. morelliforme }\end{array}$ & $\begin{array}{l}\text { Chimaltenango, Huehuetenango, } \\
\text { Quetzaltenango, Sololá }\end{array}$ \\
\hline $\begin{array}{l}\text { J. G. Hawkes, } \\
\text { J. Peter Hjerting, } \\
\text { y R. Lester }\end{array}$ & oct. 29-nov. 11, 1958 & $\begin{array}{l}\text { S. agrimonifolium, S. clarum } \\
\text { S. bulbocastanum, S. demissum, } \\
\text { S. morelliforme }\end{array}$ & $\begin{array}{l}\text { Baja Verapaz, Huehuetenango, } \\
\text { El Progreso, Quetzaltenango, } \\
\text { Quiché, Sacatepequez, } \\
\text { San Marcos, Totonicapan }\end{array}$ \\
\hline John H. Beaman & $\begin{array}{l}\text { ago. } 5-13,1959 ; \\
\text { jul. } 30 \text {-ago. } 14,1960\end{array}$ & $\begin{array}{l}\text { S. agrimonifolium, S. clarum, } \\
\text { S. demissum }\end{array}$ & $\begin{array}{l}\text { Huehuetenango, San Marcos, } \\
\text { Totonicapan }\end{array}$ \\
\hline $\begin{array}{l}\text { Keit E. Roe, E. Roe } \\
\text { y Scott A. Morí }\end{array}$ & jul. 31,1965 & S. bulbocastanum & Quetzaltenango \\
\hline
\end{tabular}




\section{MATERIALES Y METODOS}

La recolección se efectuó durante los meses de setiembre, octubre y noviembre de 1995. La ruta seguida se presenta en la Figura 1, la cual se basó en la información previamente obtenida de: 1) base de datos del CGN (Hoekstra y Seidewitz, 1987) y NRSP-6 (Bamberg y Martín, 1993); 2) literatura de Correll (1962), quien obtuvo los datos de especímenes de varios herbarios de Estados Unidos; 3) de la inspección de los autores en algunos herbarios de Estados Unidos y los herbarios de Guatemala; 4) en datos de herbario sin publicar del Dr. Jack Hawkes.

Los viajes de recolección se hicieron por medio de vehículo, sin embargo, para llegar a varias localidades hubo necesidad de caminar por uno o dos días, o bien hacer recorridos de hasta 1,000 m o más de distancia vertical.

En cada sitio de recolecta se tomaron muestras de herbario y se recolectaron frutos (con excepción de S. demissum que fue imposible encontrar frutos y se recolectó tubérculos). Después se extrajo la semilla y ya seca fue colocada en bolsas de papel. En cada sitio se tomaron datos de latitud y longitud por medio de un "Global Positioning System" (GPS) y la altura por medio de un altímetro. A nivel de gabinete para la localización de los puntos de recolección se utilizó la información obtenida

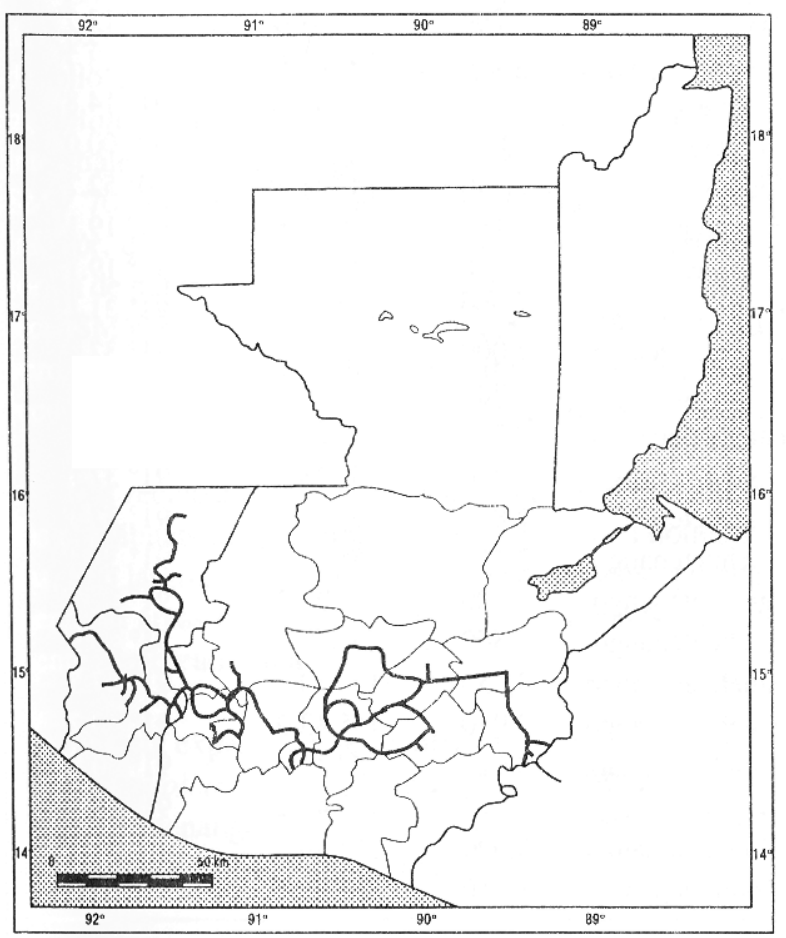

Fig. 1. Mapa de Guatemala presentando la ruta seguida en la recolección de papa silvestre, septiembre-octubre 1995. de: 1) Diccionario Geográfico de Guatemala (Gall, 1978, 1981, 1983a,b), 2) Gazette of Guatemala (United States Department of Interior, 1984); 3) mapas topográficos escala 1:50,000 (259hojas), mapas topográficosescala 1:250,000 (13 hojas) y mapa vial turístico escala 1:1,000,000 del Instituto Geográfico Militar.

Un juego completo de vaucher de herbario fueron depositados en el herbario de la Facultad de Agronomía de la Universidad de San Carlos (AGUAT), en el herbario PTIS de Holanda, y W AG de Winscousin, y algunos en BIGUAde la Escuela de Biología de la Universidad de San Carlos. Las recolecciones con los números del 7004 al 7053 fueron abreviadas como SMH, y las de los números del 7054 al 7076, abreviadas como SMV. Por acuerdo escrito con el ICT A, toda la semilla fue tomada para CGN y NRSP-6 para el incremento de la misma y en el año 1996 seráenviada una copia a Guatemala para su conservación.

\section{RESULTADOS Y DISCUSION}

En el Cuadro 2 se presenta la lista de especies recolectadas con los principales datos de pasaporte.

En total se obtuvieron 43 recolectas (13 de S. agrimonifolium, seis de S. bulbocastanum, 17 de S. clarum, una de $S$. demissum y seis de $S$. morelliforme), un resumen representativo de germoplasmade las especies silvestres de papa de Guatemala, como resultado de esta recolecta se presenta en el Cuadro 3.

\section{Solanum agrimonifolium}

La especie $S$. agrimonifolium posee hojas compuestas con un largo de $40 \mathrm{~cm}$ o más, con 7 a 9 pares de foliolos laterales y cada uno con 20 pares de foliolos intersticiales, flores con corola de color púrpura de 2,5 $\mathrm{cm}$ de diámetro, frutos cónicos apicalmente agudos. Esta es la única especie de papa silvestre de Guatemala, que aún tiene problemas en sus límites como especie con S. oxycarpum. Solanum agrimonifolium es una de las 40 especies incluidas por Hawkes (1990) en la serie Conicibaccata Bitter. De acuerdo con Hawkes (1990), las otras especies representativas de esta serie son S. oxycarpum, restringida a México; $S$. longiconicum, Bitter de Costa Rica y Panamá y $S$. woodsonii Correll de Costa Rica, Panamá y Venezuela.

Correll (1962), por otro lado, ha identificado algunas recolectas de Costa Rica y Panamá como $S$. oxycarpum y considera a $S$. longiconicum como sinonimia de $S$. oxycarpum. Gentry y Standley (1974) han determinado a 
Cuadro 2. Especies de papa silvestre (Solanum spp.) recolectadas en Guatemala, setiembre-octubre 1995.

\begin{tabular}{|c|c|c|c|c|c|c|}
\hline No. colecta & Especie & fecha & Departamento & Altitud & Latitud $\mathbf{N}$ & Longitud $\mathrm{W}$ \\
\hline SMH-7004 & S. morelliforme & $15-09-95$ & Totonicapan & 2,810 & $14^{\circ} 15.6^{\prime}$ & $91^{\circ} 20.3^{\prime}$ \\
\hline SMH-7005 & S. morelliforme & 15-09-95 & Totonicapan & 2,960 & $14^{\circ} 55.3^{\prime}$ & $91^{\circ} 19.3^{\prime}$ \\
\hline SMH-7006 & S. agrimonifolium & $15-09-95$ & Totonicapan & 3,150 & $14^{\circ} 54.8^{\prime}$ & $91^{\circ} 19.1^{\prime}$ \\
\hline SMH-7007 & S. clarum & $16-09-95$ & Huehuetenango & 3,370 & $15^{\circ} 28.5^{\prime}$ & $91^{\circ} 30.5^{\prime}$ \\
\hline SMH-7008 & $S$. sp. & $16-09-95$ & Huehuetenango & - & $15^{\circ} 33^{\circ}$ & $91^{\circ} 31^{\prime}$ \\
\hline SMH-7009 & S. morelliforme & $17-09-95$ & Huehuetenango & 3,050 & $15^{\circ} 46^{\prime}$ & $91^{\circ} 30.3^{\prime}$ \\
\hline SMH-7010 & S. bulbocastanum subsp. partitum & $18-09-95$ & Huehuetenango & 2,000 & $15^{\circ} 18^{\prime}$ & $91^{\circ} 31^{\prime}$ \\
\hline SMH-7011 & S. clarum & $20-09-95$ & Totonicapan & 3,020 & $15^{\circ} 04.3^{\prime}$ & $91^{\circ} 33.5^{\prime}$ \\
\hline SMH-7013 & S. morelliforme & $20-09-95$ & Totonicapan & 3,010 & $15^{\circ} 03.8^{\prime}$ & $91^{\circ} 33.9^{\prime}$ \\
\hline SMH-7014 & S. clarum & $20-09-95$ & Totonicapan & 3,010 & $15^{\circ} 03.8^{\prime}$ & $91^{\circ} 33.9^{\prime}$ \\
\hline SMH-7019 & S. agrimonifolium & $22-09-95$ & Quetzaltenango & 2,900 & $14^{\circ} 45^{\prime}$ & $91^{\circ} 25^{\prime}$ \\
\hline SMH-7021 & S. agrimonifolium & $23-09-95$ & San Marcos & 2,340 & $14^{\circ} 56.8^{\prime}$ & $91^{\circ} 51.3^{\prime}$ \\
\hline SMH-7026 & S. agrimonifolium & $24-09-95$ & San Marcos & 3,380 & $15^{\circ} 10.3^{\prime}$ & $91^{\circ} 56.8^{\prime}$ \\
\hline SMH-7027 & S. clarum & $24-09-95$ & San Marcos & 3,380 & $15^{\circ} 10.3^{\prime}$ & $91^{\circ} 56.8^{\prime}$ \\
\hline SMH-7028 & S. clarum & 24-09-95 & San Marcos & 3,360 & $15^{\circ} 10.3^{\prime}$ & $91^{\circ} 58.4^{\prime}$ \\
\hline SMH-7029 & S. clarum & $25-09-95$ & San Marcos & 3,260 & $15^{\circ} 11^{\prime}$ & $92^{\circ} 04^{\prime}$ \\
\hline SMH-7030 & S. morelliforme & $25-09-95$ & San Marcos & 2,900 & $15^{\circ} 08^{\prime}$ & $92^{\circ} 07^{\prime}$ \\
\hline SMH-7034 & S. agrimonifolium & $26-09-95$ & San Marcos & 2,780 & $15^{\circ} 046^{\prime}$ & $91^{\circ} 52.8^{\prime}$ \\
\hline SMH-7036 & S. agrimonifolium & $28-09-95$ & Sololá & 3,000 & $14^{\circ} 51.5$ & $91^{\circ} 11.7^{\prime}$ \\
\hline SMH-7040 & S. bulbocastanum subsp. partitum & $05-10-95$ & Baja Verapaz & 1,430 & $15^{\circ} 09.3^{\prime}$ & $90^{\circ} 17.5^{\circ}$ \\
\hline SMH-7042 & S. bulbocastanum subsp. parititum & $05-10-95$ & Baja Verapaz. & 1,640 & $15^{\circ} 10.3^{\prime}$ & $90^{\circ} 17.7^{\prime}$ \\
\hline SMH-7043 & S. bulbocastanum subsp. partitum & $05-10-95$ & Baja Verapaz & 1,420 & $15^{\circ} 12.9^{\prime}$ & $90^{\circ} 17.7$ \\
\hline SMH-7046 & S. clarum & $12-10-95$ & Totonicapan & 3,260 & $14^{\circ} 51.6^{\prime}$ & $91^{\circ} 13.7^{\prime}$ \\
\hline SMH-7048 & S. clarum & $12-10-95$ & Totonicapan & 3,220 & $14^{\circ} 52.6^{\prime}$ & $91^{\circ} 13.7^{\prime}$ \\
\hline SMH-7049 & S. clarum & $12-10-95$ & Totonicapan & 3,220 & $14^{\circ} 52.6^{\prime}$ & $91^{\circ} 14.8^{\prime}$ \\
\hline SMH -7050 & S. agrimonifolium & $13-10.95$ & Chimaltenango & 2.880 & $14^{\circ} 00.0^{\prime}$ & $90^{\circ} 00.0^{\prime}$ \\
\hline SMH-7051 & S. agrimonifolitim & $13-10.95$ & Chimaltenango & 2,840 & $14^{\circ} 31.6^{\circ}$ & $90^{\circ} 52.7^{\prime}$ \\
\hline SMV-7054 & S. demissum & $17-10-95$ & Totonicapan & 3,180 & $14^{\circ} 54.8^{\prime}$ & $91^{\circ} 19.1^{\prime}$ \\
\hline SMV-7055 & S. clarum & $17-10-95$ & Totonicapan & 3,180 & $14^{\circ} 54.8^{\prime}$ & $91^{\circ} 19.1^{\prime}$ \\
\hline SMV-7056 & S. buibocastanum subsp. partitum & $18-10-95$ & Huehuetenango & 1,900 & $15^{\circ} 18.8^{\prime}$ & $91^{\circ} 31.1^{\prime}$ \\
\hline SMV -7057 & S. bulbocastanum subsp. partitum & $19-10-95$ & Huehuetenango & 2,000 & $15^{\circ} 19.3^{\prime}$ & $91^{\circ} 32.9^{\prime}$ \\
\hline SMV-7059 & S. clarum & $20-10-95$ & Huehuetenango & 3,280 & $15^{\circ} 27.6^{\prime}$ & $91^{\circ} 31.2^{\prime}$ \\
\hline SMV-7061 & S. agrimonifolium & $23-10-95$ & Huehuetenango & 2,730 & $15^{\circ} 28.6^{\prime}$ & $91^{\circ} 37.4^{\prime}$ \\
\hline SMV-7062 & S. agrimonifolium & $23-10-95$ & Huehuetenango & 2,830 & $15^{\circ} 28.7^{\prime}$ & $91^{\circ} 37.3^{\prime}$ \\
\hline SMV-7063 & S. agrimonifolium & $23-10-95$ & Huehuetenango & 2,880 & $15^{\circ} 28.9^{\prime}$ & $91^{\circ} 37.3^{\prime}$ \\
\hline SMV-7064 & S. clarum & $24-10-95$ & Huehuetenango & 3,430 & $15^{\circ} 29.2^{\prime}$ & $91^{\circ} 30.4^{\prime}$ \\
\hline SMV-7065 & S. clarum & $24-10-95$ & Huehuetenango & 3,500 & $15^{\circ} 29.2^{\prime}$ & $91^{\circ} 30.9^{\prime}$ \\
\hline SMV-7066 & S. clarum & $24-10-95$ & Huehuetenango & 3,500 & $15^{\circ} 28.5^{\prime}$ & $91^{\circ} 30.9^{\prime}$ \\
\hline SMV-7067 & S. clarum & $25-10-95$ & Huehuetenango & 3,350 & $15^{\circ} 30.1^{\prime}$ & $91^{\circ} 27.5^{\prime}$ \\
\hline SMV-7069 & S. morelliforme & $26-10-95$ & Huehuetenango & 2,930 & $15^{\circ} 43.9^{\prime}$ & $91^{\circ} 30.2^{\prime}$ \\
\hline SMV-7072 & S. clarum & $26-10-95$ & Huehuetenango & 3,020 & $15^{\circ} 49.2^{\prime}$ & $91^{\circ} 30.5^{\prime}$ \\
\hline SMV-7073 & S. agrimonifolium & $25-10-95$ & Huehuetenango & 3,020 & $15^{\circ} 49.2^{\prime}$ & $91^{\circ} 30.5^{\prime}$ \\
\hline SMV-7074 & S. clarum & $26-10-95$ & Huehuetenango & 3,020 & $15^{\circ} 49.2^{\prime}$ & $91^{\circ} 30.5^{\prime}$ \\
\hline SMV-7075 & S. clarum & $27-10-95$ & Totonicapan & 3,150 & $14^{\circ} 54.4^{\prime}$ & $91^{\circ} 17.7^{\prime}$ \\
\hline
\end{tabular}


Cuadro 3. Número y lugar de recolección del germoplasma de las cinco especies de papa silvestres de Guatemala.

\begin{tabular}{|c|c|c|c|}
\hline Especie & $\begin{array}{l}\text { Accesiones de, } \\
\text { México antes de } \\
\text { esta expedición }{ }^{1}\end{array}$ & $\begin{array}{l}\text { Accesiones de, Guatemala } \\
\text { por departamento, antes } \\
\text { de esta expedición }\end{array}$ & $\begin{array}{l}\text { Total de accesiones de, Gua- } \\
\text { temala por departamento, } \\
\text { después de esta expedición }\end{array}$ \\
\hline Solanum agrimonifolium & 3 & $6: 3(\mathrm{H}), 1(\mathrm{SMH}), 1(\mathrm{~T})$ & $\begin{array}{l}\text { 19:2(C), 8(H), 2(Qe), } 4(\mathrm{SMH}) \\
\text { 1(So), } 2(\mathrm{~T})\end{array}$ \\
\hline S. bulbocastanum & 40 & $1: 1(\mathrm{H})$ & 7: 3(B), 4(H) \\
\hline S. clarum & 1 & $4: 1(\mathrm{So}), 3(\mathrm{~T})$ & $21: 7(\mathrm{H}), 3(\mathrm{SMH}), 1(\mathrm{So}), 10(\mathrm{~T})$ \\
\hline S. demissum & 140 & $1: 1(\mathrm{H})$ & 2: $1(\mathrm{H}) 1(\mathrm{~T})$ \\
\hline S. morelliforme & 8 & 4: 1(C), 2(Qe), 1(Qi) & 10: 1(Qe), 2(H), 1(Qi), 3(T) \\
\hline
\end{tabular}

${ }^{1}$ Baja Verapaz (B), Chimaltenango (C), Guatemala (G), Huehuetenango (H), El Progreso (P), Quetzaltenango (Qe), Quiché (Qi), San Marcos (SMH), Sacatepequez (Sa), Sololá (So), Totonicapan (T).

la accesión Steyermak 43672 (espécimen en F) del departamento de El Progreso, Guatemala, como $S$. oxycarpum, un espécimen recolectado después por Correll (1962), y no comentado por Hawkes (1990).

Esta especie es considerada como distinta de $S$. agrimonifolium por el número y tamaño los folio los laterales e interstiales y por la pubescencia de la hoja (Correll, 1962; Gentry y Standley, 1974; Hawkes, 1990).

En esta recolección se encontró una posible población de S. oxycarpus (SMV 7073) que tiene un número reducido de foliolos interstiales, creciendo con plantas típicas de S. agrimonifolium (SMV 7074) con muchos foliolos interstiales, y con fenotipos intermedios. $\mathrm{Cu}-$ riosamente, SMV 7073 tiene frutos cónicos redondeados en su parte inferior, diferentes a los típicos, con la parte inferior en forma de punta de S. agrimonifolium (incluyendo la recolecta SMV 7074). Sin embargo, mientras se determina su verdadera identidad, para los propósitos de este artículo, la recolecta SMV 7073 se considera como $S$. agrimonifolium.

Las recolectas de S. agrimonifolium fueron realizadas en hábitats muy húmedos y disturbados, en hondonadas, a lo largo de arroyos, con suelos orgánicos, generalmente en áreas de bosques nubosos,y en lugares parcialmente sombreados o a pleno sol.

En esta expedición se recolectaron 13 accesiones de S.agrimonifolium en los departamentos de Huehuetenango, Quetzaltenango, Totonicapan, Sololá y Chimaltenango en altitudes de 2,340 a 3,380 msnm.

\section{Solanum bulbocastanum}

La especie Solanum bulbocastanum posee tallos pubescentes, hojas simples pubescentes de forma ova- lada, y todas las muestras recolectadas en esta expedición tienen los lóbulos de la corola profundamente divididos, por lo que corresponden a la subsp. partitum (Correll) Hawkes (Correll, 1962; Gentry y Standley, 1974; Hawkes, 1990). Esta subespecie, a diferencia de las otras especies silvestres de papa de Guatemala, usualmente crecen en lugares a baja altitud (1,350-2300 msnm) y a menudo en hábitats secos. Persiste en áreas dedicadas al pastoreo, y es muy apetecida por el ganado bovino antes de que florezca y fructifique. Todas las recolectas de fruto en tales áreas fueron realizadas en los lugares de más difícil acceso para los animales, tales como pendientes con rocas, paredes de rocas o entre arbustos espinosos o cactos. Es una planta inconspicua con una amplia y dispersa distribución a través de las áreas secas de Guatemala.

En esta investigación se recolectaron seis accesiones en los departamentos de Baja Verapaz y Huehuetenango en altitudes de 1,430 a 2,000 msnm.

\section{Solanum clarum}

La especie Solanum clarum posee hojas cordadas simples y glabras, flores con los lóbulos de la corola de color purpura en la punta y blanco hacia la base. Solanum clarum y $S$. morelliforme fueron separadas por Hawkes (1990) en la serie Bulbocastana (Rydb.) Hawkes la primera (también contiene a $S$. bulbocastanum) y en la monotípica serie Morelliformia Hawkes la segun$\mathrm{da}$, con base en las diferencias florales, reproductivas, serológicas y ecológicas (serie Bulbocastanum terrestre, serie Morelliformia epífita). Basado en los datos del ADN del cloroplasto, sin embargo, S. clarum y S. morelliforme están unidos como taxa hermanos, y S. bulbocastanum y $S$. clarum divididos dentro de distintos grupos (Spooner y Sytsma, 1992). Otros caracteres reproductivos, citológicos y morfológicos suponen a $S$. clarum y $S$. morelliforme como taxa hermanos como lo 
indican Spooner y Sytsma (1992). En la presente investigación se encontró a $S$. clarum comúnmente creciendo en el suelo en lugares con musgo, en la sombra en condiciones parecidas a las de una epífita, (aunque ocasionalmente también como una epífita).

Solanum clarum crece en lugares con bosques de pino y/o pinabete, usualmente en asociación con Acaena elongata L., Alchemilla pectinata HBK, y Pemetta ciliata (Schlecht. y Cham.) Small. Muchas poblaciones de $S$. clarum consisten en cientos de plantas en diferentes estados de crecimiento; sin embargo, las flores fueron raramente vistas, y los frutos generalmente difíciles de localizar, sin embargo, sí fue posible obtener frutos maduros de todas las poblaciones encontradas.

De $S$. clarum, se obtuvieron 17 recolectas en los departamentos de Huehuetenango, San Marcos y Totonicapan en altitudes de 3,010 a 3,380 msnm.

\section{Solanum demissum}

La especie Solanum demissum que crece en forma de roseta y en ocasiones es ascendente; los foliolos son sésiles, redondeados y obtusos en el ápice, corola purpura generalmente con lóbulos muy cortos. Esta especie tiene autopolinización, y produce fácilmente frutos en condiciones naturales. Es una de las especies más comunes de papa silvestre en los bancos de germoplasma. Sin embargo, todas las recolectas que se tienen pertenecen del norte y sur de México, donde está ampliamente distribuida, y no se tiene de Guatemala donde es una especie rara. Ha sido consignada en los departamentos de Huehuetenango, Sacatepéquez y Totonicapan, pero en esta investigación se obtuvo sólo una recolecta en Totonicapan a una altitud de 3,180 msnm, que carecía de flores y frutos por lo que es la única de la cual fue preciso recolectar tubérculos.

Hawkes (1990) incluye a $S$. demissum en la serie Demissa Buk., junto con otras cinco especies hexaploides de México. Las características morfológicas definidas de esta serie no son claras, sin embargo, Spooner, Den Berg, Bamberg (1995) indica que sobre la base de la morfología, la especie es más similar a $S$. albicans (sección Acaulia Jus.) de Suramérica que a cualquier otra especie de la serie Demissa.

\section{Solanum morelliforme}

La especie Solanum morelliforme es de hojas simples, flor con corola blanca, pequeña y en forma estrellada. Esta especie es el único miembro de la sección Petota que crece casi exclusivamente como una epífita ocasionalmente. Sin embargo, la especie es encontrada en el suelo sobre madera en descomposición, en raíces de árboles caídos o sobre musgo. Todas las poblaciones fueron recolectadas en condiciones sombreadas, en la inserción del tallo principal con ramas gruesas, en árboles maduros de aliso, pino o encino. Fue imposible localizar la especie en localidades previamente documentadas debido a que habían sido deforestadas. Debido a que los frutos y las semillas de todos los miembros de la sección Petota no se distribuyen por el viento, la especie probablemente es distribuida por pájaros o por animales que viven en los árboles.

De $S$. morelliforme, se recolectaron seis accesiones en los departamentos de Huehuetenango, San Marcos y Totonicapan en alturas comprendidas de 2,810 a 3,050 msnm.

La recolección se enfocó a las áreas con localidades previamente recolectadas, pero carentes de germoplasma para maximizar el trabajo. Adicionalmente, se buscó en áreas con elevaciones apropiadas para el crecimiento de papas silvestres, para las cuales no se tenían datos de recolección. Estas incluyeron montañas en los departamentos de Guatemala, Jalapa y Chimaltenango con elevaciones de 2,000 a 3,000 msnm. A pesar que estas localidades tienen sitios relativamente poco disturbados con vegetación con muchas epífitas aparentemente es ideal para el crecimiento de S. agrimonifolium y S. morelliforme, no fue posible encontrar papas silvestres en el lugar.

En esta investigación se encontró sólo algunas de las poblaciones conocidas (Correll, 1962); muchas de ellas están ahora aparentemente extintas debido la destrucción de su hábitat. Según se puede verificar, mucho del bosque primario ha sido destruido por incendios, por daño del gorgojo del pino (Dendroctonus spp.), por tala para uso de la madera para construcción o como combustible, o tala para utilizar el suelo como área agrícola. En muchos lugares, los bosques que albergaban papas silvestres están ahora reducidos a pequeñas áreas aisladas, rodeadas por campos clareados carentes de éstos.

El futuro de estas especies es bastante incierto, si se toma en consideración, que actualmente Guatemala tiene una población de 10 millones de habitantes, de los cuales el 62\% es rural (UNICEF, 1994), que ejerce una gran presión sobre el recurso bosque; lo que se ve reflejado en el aumento de la deforestación que entre 1978 a 1988 ha tenido un promedio de 60,000 ha por año, (Dirección General de Bosque y Vida Silvestre, 1994), con un $33 \%$ en los bosques donde las especies silvestres de papa se desarrollan. A esto debe agregarse el sobrepastoreo que existe en muchas de estas áreas. Pareciera entonces que en un alto porcentaje la sobrevivencia de estas especies va a depender del 
manejo adecuado que se le preste a las áreas protegidas existentes actualmente y de las que llenen esta categoría en el futuro, si se toma en cuenta los lugares donde crecen estas especies. Así, Solanum morelliforme crece sobre árboles maduros, y es probablemente la más susceptible a desaparecer por la deforestación. Solanum clarum y S. demissum usualmente crecen sólo en lugares sombreados bajo árboles maduros entre musgo u hojarasca de pino, y son rápidamente comidas por el ganado (ovejas y cabras especialmente), y $S$. demissum es ahora muy rara en Guatemala. S. agrimonifolium crece en hábitats mucho más desordenados, pero casi siempre en áreas recientemente clareadas de bosques primarios en suelos profundos, orgánicos y húmedos, y también es muy gustado por el ganado. S. bulbocastanum que crece entre arbustos de áreas secas de pastoreo, y algunas de las recolectas realizadas en esta investigación, puede ser que sea la última vez que se obtengan en estos lugares.

\section{CONCLUSIONES}

Las 43 nuevas recolectas efectuadas en esta investigación y las 16 previas de Graham y Hawkes, proveen una buena representatividad para todas las especies de papa silvestre presentes en Guatemala (a excepción de S. demissum), considerando las poblaciones actualmente existentes y la cobertura geográfica obtenida.

Consecuentemente, futuras recolecciones de germoplasma de papa silvestre deben ser conducidas en países menos recolectados, tales como Costa Rica, Honduras y Panamá, para tener la distribución geográfica de estas especies a nivel de Centroamérica.

Sin embargo, si una nueva recolección de papas silvestres fuera planificada para Guatemala, se sugieren las siguientes áreas que no fueron posibles de explorar en la presente recolección:

1. La inestabilidad política existente no permitió explorar los volcanes que se encuentran alrededor dcl lago de Atitlán en el departamento de Sololá con elevaciones de 2,995 a 3,537 msnm (volcanes de Atitlán, San Pedro y Tolimán). No se tienen consignados registros de estos lugares, pero estas son probablemente áreas ricas para las cinco especies que existen en Guatemala. Así mismo, la parte este de la Sierra de los Cuchumatanes en el departamento de El Quiché, hacia el norte por el camino de Río Blanco a Uspantán, con algunas elevaciones arriba de los 2,700 msnm.

2. Las montañas en la porción del extremo suroeste de la Sierra de los Cuchumatanes, en el departa- mento de Huehuetenango, al norte de Cuilco, y el extremo de la parte norte de esta sierra al norte de San Mateo Ixtatán.

3. La Sierra de la Minas en su parte este en el departamento de El Progreso, y en la parte oeste en el departamento de Zacapa. Hay muchos hábitats apropiados especialmente para $S$. agrimonifolium o para $S$. oxycarpum donde se muestreo, y probablemente hay otros similares en las cercanías.

4. Solanum bulbocastanum crece en sitios más secos que el de las otras especies de papa silvestre de Guatemala. La especie probablemente es poco recolectada debido a que es inconspicua, por lo que pasa desapercibida. En Guatemala la especie crece en alturas de 1,350 a 2,300 msnm, por lo que es probable encontrarla en otras áreas de Guatemala con estas condiciones.

Con base en la experiencia de este viaje en cuanto a la fructificación de las especies de papa silvestre, y tomando en consideración que se puede obteneruna mayor eficiencia de recolección en época seca, se sugiere que el mejor tiempo para la recolección de frutos con semillas de papa silvestre en Guatemala podría ser durante los meses de octubre y noviembre.

Dos áreas son notables por la concentración de especies silvestres de papa y porque aún mantienen determinadas áreas casi naturales y pueden proponerse como zonas de conservación in situ: 1) La Cumbre de María Tecún en el departamento de Totonicapan, y 2) áreas dispersas en la Sierra de los Cuchumatanes. Ambas áreas contienen a todas las poblaciones de papa silvestre consignadas para Guatemala excepto S. bulbocastanum, además estas áreas son mencionadas en la Ley de Áreas Protegidas de Guatemala (Decreto 4-89) como oficialmente protegidas bajo esta ley, sin embargo, se carece de estudios técnicos formales de ambas para que en la práctica se les de un manejo como áreas protegidas.

\section{LITERATURA CITADA}

BAMBERG, J.B; MARTIN, M.W. 1993. Inventory oftuberbearning Solanum species. Potato introduction station. NRSP-6. Sturgeon Bay, Wisconsin.

BAMBERG, J.B; SPOONER, D.M. 1994. The United States potato introduction station herbarium. Taxon.43:489496.

CORRELL, D.S. 1952. Section Tuberarium of the genus Solanum of North America and Central America. U.S.D.A. Agric. Monogr. 11:1-243. 
CORREL, D.S. 1962. The potato and its wild relatives. Contrib. Texas Res. Found. Bot. Stud. 4: 1-606.

DIRECCION GENERAL DE BOSQUES Y VIDA SIL VESTRE. 1994. La de1'orestación en Guatemala: causas y soluciones. Ministerio de Agricultura Ganadería y Alimentación. Guatemala.

GALL, F. 1978. Diccionario geográfico nacional. $2^{\mathrm{a}}$ ed. Tomo 1. A-Ch. Instituto Geográfico Nacional. Guatemala. Guatemala.

GALL, F. 1981. Diccionario geográfico nacional. $2^{\mathrm{a}} \mathrm{ed}$. Tomo 11. D-P. Instituto Geográfico Nacional. Guatemala. Guatemala.

GALL, F. 1983a. Diccionario geográfico nacional. 2a ed. Tomo 111. Q-S. Instituto Geográfico Nacional. Guatemala. Guatemala.

GALL, F. 1983b. Diccionario geográfico nacional. $2^{\mathrm{a}}$ ed. Tomo IV. T-Z. Instituto Geográfico Nacional. Guatemala. Guatemala.

GENTRY,J.L;STANDLEY,P.C. 1974. Flora of Guatemala $\mathrm{X}$ :Solanaceae. Fieldiana, Bot. 24 (1,2):1-151.

GRAHAM, K.M.; NEIDERHAUSER, J.S; SERVIN, L. 1959. Studies of fertility and late blight resistence in Solanum bulbocastanum Dun. in Mexico. Canad. J. Bot. 37:41-49.

GRAHAM, K.M.; L.A. DIONNE. 1961. Crossability relationships of certain diploid Mexican Solanum species. Canad. J. Genet. Cytol. 3:121-127.
HAWKES, J.G. 1959. The Birmingham University plant coIlecting expedition to Mexico and Central America. Univ. Birmingham Gaz. 11:60-63.

HAWKES, J.G. 1990. The potato:evolution, biodiversity and genetic resources. Smithsonian Institution Press. Washington, D.C. 259 p.

HOEKSTRA, R.; L. SEIDEWITZ. 1987. Evaluation data on tuberbearing Solanum species. Second edition. Dutch German Curatorium for plant genetic resources. Braunschweig.

SPOONER, D.M.; K.J. SYTSMA. 1992. Examinationo1'series relationships of Mexican and Central American wild potatoes (Solanum sect. Petota): evidence from chloroplast DNA restriction site variation. Syst. Bot. $17: 432-448$.

SPOONER, D.M.; R.G. van DEN BERG ; J.B. BAMBERG. 1995. Examination of species boundaries of Solanum series Demissa y potentially related species in series Acaulia an series Tuberosa (sect. Petota). Syst. Bot. 20:295-314.

UNICEF. 1994. Realidad socioeconómica de Guatemala: con énfasis en la situación del niño y la mujer. UNICEF: SEGEPLAN. Piedra Santa. Guatemala.

UNITED STA TES DEP ART AMENT OF INTERIOR. 1984. Gazette of Guatemala: official standard names approved by the U.S. Board on Geographic Names. Division of Geography. Departament of Interior, U.S. Government. Washington, D.C. 